\title{
BMJ Open Risk of active tuberculosis in migrants diagnosed with cancer: a retrospective cohort study in British Columbia, Canada
}

\author{
Divjot S Kumar (D) , ${ }^{1}$ Lisa A Ronald, ${ }^{2}$ Kamila Romanowski, ${ }^{2,3}$ Caren Rose, ${ }^{2}$ \\ Hennady P Shulha, ${ }^{2}$ Victoria J Cook, ${ }^{1,2}$ James C Johnston ${ }^{1,2}$
}

To cite: Kumar DS, Ronald LA, Romanowski K, et al. Risk of active tuberculosis in migrants diagnosed with cancer: a retrospective cohort study in British Columbia, Canada. BMJ Open 2021;11:e037827. doi:10.1136/ bmjopen-2020-037827

- Prepublication history and additional material for this paper is available online. To view these files, please visit the journal online (http://dx.doi.org/10. 1136/bmjopen-2020-037827)

Received 24 February 2020 Revised 15 January 2021 Accepted 24 January 2021

Check for updates

(C) Author(s) (or their employer(s)) 2021. Re-use permitted under CC BY-NC. No commercial re-use. See rights and permissions. Published by BMJ.

${ }^{1}$ Division of Respiratory Medicine, Faculty of Medicine, University of British Columbia, Vancouver, British Columbia, Canada

${ }^{2}$ British Columbia Centre for Disease Control, Vancouver, British Columbia, Canada

${ }^{3}$ Department of Medicine, University of British Columbia, Vancouver, British Columbia, Canada

Correspondence to

Dr James C Johnston;

James.Johnston@bccdc.ca

\section{ABSTRACT}

Objectives To describe the association between types of cancer and active tuberculosis (TB) risk in migrants. Additionally, in order to better inform latent TB infection (LTBI) screening protocols, we assessed proportion of active TB cases potentially preventable through LTBI screening and treatment in migrants with cancer. Design Population-based, retrospective cohort study. Setting British Columbia (BC), Canada.

Participants 1000764 individuals who immigrated to Canada from 1985 to 2012 and established residency in BC at any point up to 2015.

Primary and secondary outcome measures Using linked health administrative databases and disease registries, data on demographics, comorbidities, cancer type, TB exposure and active TB diagnosis were extracted. Primary outcomes included: time to first active TB diagnoses, and risks of active TB following cancer diagnoses which were estimated using Cox extended hazard regression models. Potentially preventable TB was defined as active TB diagnosed $>6$ months postcancer diagnoses.

Results Active TB risk was increased in migrants with cancer ((HR $(95 \% \mathrm{Cl})) 2.5(2.0$ to 3.1$))$, after adjustment for age, sex, TB incidence in country of origin, immigration classification, contact status and comorbidities. Highest risk was observed with lung cancer (HR 11.2 (7.4 to 16.9)) and sarcoma (HR 8.1 (3.3 to 19.5)), followed by leukaemia (HR 5.6 (3.1 to 10.2)), lymphoma (HR 4.9 (2.7 to 8.7)) and gastrointestinal cancers (HR 2.7 (1.7 to 4.4)). The majority $(65.9 \%)$ of active TB cases were diagnosed $>6$ months postcancer diagnosis.

Conclusion Specific cancers increase active TB risk to varying degrees in the migrant population of $\mathrm{BC}$, with approximately two-thirds of active TB cases identified as potentially preventable.

\section{INTRODUCTION}

Tuberculosis (TB) remains the top single infectious disease cause of death worldwide, with an estimated 10 million cases and 1.4 million deaths in 2018. ${ }^{1}$ Recognising this, WHO created the EndTB strategy with a vision of global TB elimination. ${ }^{2}$ A core part of the EndTB strategy is identification and
Strengths and limitations of this study

- Our study included a large cohort with $>1$ million people over 19-year period.

- First study to assess risk of active tuberculosis (TB) among different malignancies in an immigrant population, and include other common malignancies such as prostate, breast and gastrointestinal cancers.

- Risk of active TB among different malignancies were adjusted for other commonly recognised TB risk factors.

- Unable to account for impact of smoking, alcohol use and chemotherapy.

- Unable to account for TB contact outside of British Columbia.

treatment of latent tuberculosis infection (LTBI) in regions with low TB incidence..$^{2-5}$ In many low incidence regions, such as Canada, efforts to meet EndTB elimination goals have been hindered by high TB rates in specific populations.${ }^{5-7}$ People that migrate to Canada, in particular, experience rates of TB over 20 times greater than the Canadian-born non-Indigenous population. ${ }^{28}$ There are also clear links between certain comorbidities and TB risk. ${ }^{8}$ New strategies to identify and treat people at highest risk for $\mathrm{TB}$ are required to accelerate TB elimination in Canada.

The association between cancer and TB risk is increasingly recognised in peer-reviewed literature and national $\mathrm{TB}$ guidelines. At present, Canadian and American guidelines recognise haematological and head and neck (HN) malignancies as risk factors for $\mathrm{TB},{ }^{89}$ and a recent systematic review and meta-analysis conducted by Cheng et $a l^{10}$ identified up to a ninefold increased risk of TB among patients with haematological, HN and lung cancers. In migrant populations, the risk of active TB associated with different 
malignancies has not been well studied and may represent an additive risk. Furthermore, literature is limited on TB risk in people with many common malignancies such as gastrointestinal (GI), breast, prostate and gynaecological (GYN) cancers.

In this study, we used linked health administrative data on migrants to the province of British Columbia (BC), Canada to describe the association between different malignancies and active TB risk within foreign born individuals. Furthermore, we aimed to assess the proportion of immigrants with cancer in whom active TB was potentially preventable through LTBI screening and treatment.

\section{METHODOLOGY}

\section{Study setting and data sources}

This study is part of a larger project ${ }^{11}$ that describes TB epidemiology in people that migrate to BC, a Canadian province with a population of 5.0 million people and TB incidence of 4.7 per 100000 persons. ${ }^{12}{ }^{13}$ In 2016, approximately $22 \%$ of the population of BC, and $85 \%$ of people diagnosed with active TB, were born outside of Canada. ${ }^{14}$

Descriptions of the databases, linkages and methods to identify the cohort have been described in detail in previous publication. ${ }^{11}$ Briefly, data were extracted from several linked health administrative databases, including a national immigration database, a provincial health insurance registry, hospitalisation, physician billing, outpatient pharmacy, vital statistics, HIV and end-stage renal disease registries. ${ }^{15-27}$ Extracted data were provided as deidentifiable datasets linked using unique scrambled identification numbers. Full details on this linkage are described in a protocol publication. ${ }^{11}$

TB diagnosis and treatment data were obtained from the $\mathrm{BC}$ Provincial $\mathrm{TB}$ registry, housed at the $\mathrm{BC}$ Centre for Disease Control (BCCDC). The BCCDC is responsible for treatment of all people with active TB and LTBI in the province, and maintains a provincial TB registry that includes data on all people with a history of TB contact, LTBI and active TB diagnosis, and treatment. ${ }^{25}$ Data on cancer diagnosis were obtained from the BC Cancer Agency (BCCA) registry. The BCCA is a government organisation that provides comprehensive cancer care to the people of $\mathrm{BC}$ in partnership with regional health authorities. The BCCA registry houses data on all primary cancers diagnosed in BC, with diagnoses confirmed based on pathology, cytology, lab results, cancer centre admissions, and death certificates. ${ }^{26}$

\section{Study population and follow-up}

Our source population included all foreign-born individuals that obtained permanent residency in Canada between 1 January 1985 and 31 December 2012 and established residency in $\mathrm{BC}$ at any point until 31 December 2015. BC residents were identified when they registered in the provincial Medical Services Plan (MSP), a universal health insurance programme administered by the BC government. Depending on a household's income, MSP coverage may be free, or may require monthly premiums. Thus, we believe that MSP coverage may be a surrogate marker of residency status in BC. ${ }^{28}{ }^{29}$ Individuals who did not acquire an MSP number (eg, temporary visitors, students, short-term workers or undocumented residents of $\mathrm{BC}$ ) were not included in the cohort.

We limited the cohort to 1996 onwards when community pharmacy data became available. Furthermore, we limited the analysis cohort to individuals aged $\geq 15$ years as there were few cancer cases at younger ages. Calculation of follow-up time began for all individuals at their index event, which was identified as occurring at 90 days before first MSP registration date or at an individual's first health service record in BC, whichever date was earliest. The 90-day window was selected to account for the mandatory 90-day waiting period required for starting MSP after arrival in BC. The follow-up period ended at first active TB diagnosis, end of MSP coverage, death or end of study period (31 December 2015), whichever date was earliest.

\section{Definitions of outcome and risk factor variables}

The primary outcome measured was time to first active TB diagnosis. TB diagnosis was identified based on BCCDC $\mathrm{TB}$ registry data, and included all TB sites (ie, pulmonary and extrapulmonary $\mathrm{TB}$ ) that were either microbiologically or clinically confirmed. TB diagnosis was established in the BCCDC registry based on the Canadian TB Reporting System Guidelines. ${ }^{30}$

The exposure of interest was incident cancer diagnosis occurring after study entry. Cancers were identified based on the site of primary malignancy, as reported in the BCCA registry. This dataset encompassed cancer diagnoses between 1 January 1985 to 31 December 2014. Specific cancers identified for this analysis included: lung, breast, GI, haematological cancers (lymphoma and leukaemia), HN, genitourinary, GYN, prostate, sarcoma, skin (including melanoma and other skin cancers), neural and other/unknown cancers. For HN cancers, thyroid malignancies were excluded due to significant differences in prognosis and management compared with other $\mathrm{HN}$ malignancies.

TB incidence rate per 100000 person-years (PYs) was calculated in people with and without a diagnosis of cancer. We identified the cancer risk start date as 90 days before the recorded cancer diagnosis date, and the cancer risk end date occurring 5 years after cancer diagnosis, at which time patients who were alive and without TB contributed time to the non-cancer group. If there were multiple overlapping cancer episodes of the same type, we tied these together and counted them as one episode only, with the first cancer diagnosis date identified as the start date of the episode. We also limited our analysis to incident cancers occurring after study entry (ie, 2014 people with prevalent cancers at the index date were excluded from analyses).

Other risk variables included sex, age (10-year age categories), time since arrival in BC ( $0-5$ years and $>5$ years), immigration classification (refugee, family or economic), 
high-risk medical comorbidities and TB incidence in country of origin. ${ }^{8}$ For the TB incidence in country of origin, we used country-level WHO TB incidence data at year of arrival to Canada from 1990 to $2012 .{ }^{31}$ For years prior to 1990, we applied 1990 TB incidence rates.

For medical comorbidities, we established diagnosis dates of the following conditions: HIV, dialysis-dependent end-stage kidney disease, diabetes and medical immunesuppression (including use of tumour necrosis factor- $\alpha$ inhibitors, high-dose steroids, high-risk disease-modifying antirheumatic drugs or receipt of solid organ or bone marrow transplantation). We also identified if an individual was a known contact of a person diagnosed with active TB in BC. Disease registries were used as the gold standard for disease diagnoses, supplemented with data from health administrative databases using validated algorithms. ${ }^{11}$ Detailed definitions and exposure risk periods for each comorbidity have been described previously. ${ }^{11} 32$

\section{Statistical analysis}

Analyses were conducted in R (V.3.6.0; The R Project for Statistical Computing) and SAS/STAT (V.9.4, SAS Institute, Cary, North Carolina, USA).

We produced descriptive statistics for our study cohort at the time of cohort entry, defined by index date. Rates of active $\mathrm{TB}$ and median years from cancer diagnosis to active TB were calculated for each cancer subtype. Cancer diagnoses may occur with or after a diagnosis of active TB, and therefore, to understand the potential impact of LTBI screening and prevention among people with cancer, we plotted the time of active TB diagnoses against the time of cancer diagnoses for each cancer type. The proportion of active TB cases that were potentially preventable was then calculated as those occurring $>6$ months after cancer diagnosis. This time point was set to capture preventable TB cases amenable to LTBI testing and treatment at cancer diagnosis.

We used extended Cox regression to model the risk of active TB. We ran separate models based on site of primary malignancy. For sex-predominant cancers, we restricted models by sex (ie, breast and GYN cancer models were restricted to females; the prostate cancer model was restricted to males). We included all identified risk variables in multivariable models, determined a priori based on clinical relevance. Schoenfeld residuals were used to test extended Cox model parameters.

Model assumptions were tested through sensitivity analyses, including testing different age at risk for cancer thresholds and the time window for the cancer at-risk period. We examined whether radiotherapy during cancer treatment increased the risk of active TB, as this has previously been suspected to be a risk factor for active TB. ${ }^{33}$ As an additional sensitivity analysis, we assessed for impact of LTBI screening within our cohort. Furthermore, we conducted sensitivity analysis assessing active TB risk stratified by TB incidence of country of origin in foreign born individuals with cancer. Lastly, we provided breakdown of pulmonary/mix TB and extrapulmonary
TB among immigrants diagnosed with cancer. Results of sensitivity analyses are reported in the online supplemental appendix 1 . Incomplete data were identified as 'missing data' in results.

\section{Patient and public involvement}

Patients or public were not involved in the methodology, conduct, analyses or reporting of this research.

\section{RESULTS}

\section{Population characteristics}

Our study population included 1000764 individuals aged $\geq 15$ years, with a mean of 13.4 PY of total follow-up time per individual. Population characteristics are outlined in table 1 . Median age was 32.3 years, $48.5 \%$ were male and $58.0 \%$ migrated from countries with TB incidence $>100$ per 100000 persons. In terms of WHO regions, 54.8\% of individuals migrated from Western Pacific, $14.5 \%$ from South East Asia and 13.2\% from Europe. A small proportion of individuals $(0.6 \%)$ were contacts of people diagnosed with active TB at any time in BC.

In the study population, 29448 people $(2.9 \%)$ were diagnosed with cancer (table 2). The most frequently noted malignancies were breast $(\mathrm{n}=5918)$, GI $(\mathrm{n}=5688)$, GYN ( $n=4450)$, lung $(n=2421)$, lymphoma $(n=1252)$ and leukaemia $(n=1139)$ cancers. Table 2 provides details on cancer types. In the entire study cohort, 2585 people developed active TB, with 91 people $(3.5 \%)$ diagnosed with active TB within 5 years of a cancer diagnosis. Unadjusted active TB rates were as follows: lung cancer (525.1 per $100000 \mathrm{PY}$ ), sarcoma (174.4 per $100000 \mathrm{PY}$ ), lymphoma (268.2 per $100000 \mathrm{PY}$ ), leukaemia (284.9 per 100000 PY), GI (107.9 per 100000 PY) and HN cancer (31.3 per $100000 \mathrm{PY}$ ) (table 2). Figure 1 shows a scatter plot with timing of TB diagnosis against timing of cancer diagnosis for each cancer type.

Potentially preventable TB cases were identified as those occurring $>6$ months after the diagnosis of cancer, as these cases could be potentially amenable to LTBI screening and therapy at the time of cancer diagnosis. Among 2421 people with lung cancer, 25 people (1.0\%) were diagnosed with active TB within 5 years. Of these, nine $(42.9 \%)$ had active TB diagnosed $>6$ months after cancer diagnosis. Similarly, $8(72.7 \%)$ with leukaemia, 6 $(50 \%)$ with lymphoma and $14(82.4 \%)$ with GI cancers were diagnosed with active $\mathrm{TB}>6$ months post-cancer diagnosis (table 2). Overall, 60 out of 91 (65.9\%) diagnosed active TB cases were classified as potentially preventable.

Figure 2 shows results from multivariable Cox regression analyses by cancer type. People with lung cancer and sarcoma had the highest risk of active TB, with adjusted HRs (aHR) of 11.2 (95\% CI 7.4 to 16.9) for lung, and 8.1 (95\% CI 3.3 to 19.5) for sarcoma. This was followed by leukaemia (aHR 5.6 (95\% CI 3.1 to 10.2)), lymphoma (aHR 4.9 (95\% CI 2.7 to 8.7)) and GI cancers (aHR 2.7 (95\% CI 1.7 to 4.4$)$ ). The full multivariable Cox model for lung cancer is shown in table 3 . 
Table 1 Cohort characteristics at entry and comorbidities over study follow-up ( $n=1000764)$

\section{Cohort characteristics} Without cancer (N (\%)) Cancer* $\left.^{*} \mathbf{N}(\%)\right)$ Total (N (\%))

Age group at baseline (years)

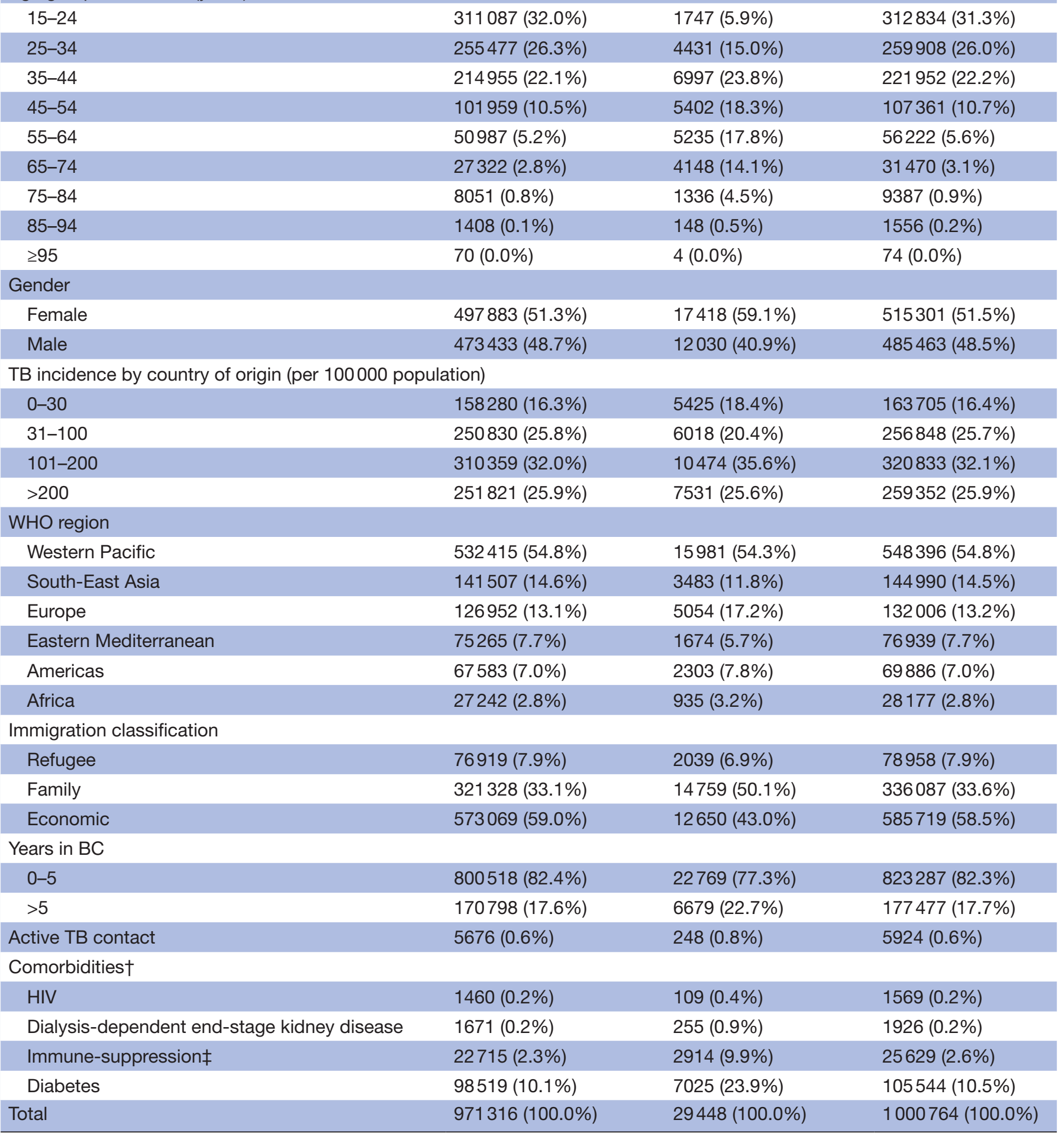

Missing data (country of origin, $n=26$, WHO region $n=370$ ).

*Includes incident cases only.

†Includes incident and prevalent cases.

łlmmune-suppression=includes treatment with immune-suppressing drugs (tumour necrosis factor- $\alpha$ inhibitors, disease-modifying antirheumatic drugs or high-dose steroids or post-transplant).

BC, British Columbia; TB, tuberculosis. 
Table 2 Incidence of active TB in patients newly diagnosed with cancer

\begin{tabular}{|c|c|c|c|c|c|c|}
\hline \multirow[b]{2}{*}{ Cancer type* } & \multirow[b]{2}{*}{ N (\%) } & \multirow[b]{2}{*}{$\begin{array}{l}\text { Median age } \\
\text { at cancer } \\
\text { diagnosis, years } \\
(Q 1, Q 3)\end{array}$} & \multirow[b]{2}{*}{$\begin{array}{l}\text { Median time } \\
\text { from cancer to } \\
\text { TB diagnosis, } \\
\text { years }(Q 1, Q 3) \dagger\end{array}$} & \multicolumn{2}{|c|}{ \# active TB diagnoses } & \multirow{2}{*}{$\begin{array}{l}\text { \# active } \\
\text { TB cases } \\
\text { per } 100000 \\
\text { person-years } \\
\text { at-risk } \ddagger\end{array}$} \\
\hline & & & & $\begin{array}{l}\text { Up to } 5 \\
\text { years after } \\
\text { cancer } \\
\text { diagnosis } \\
\end{array}$ & $\begin{array}{l}\text { From } 6 \text { months to } 5 \\
\text { years after cancer } \\
\text { diagnosis ('potentially } \\
\text { preventable TB') }\end{array}$ & \\
\hline Lung & $2421(0.2)$ & $68(57,77)$ & $0.4(0.3,0.7)$ & 25 & 9 & 525.1 \\
\hline Sarcoma & $725(0.1)$ & $47(36,58)$ & $1.6(0.7,1.9)$ & 5 & $<5$ & 174.4 \\
\hline Lymphoma & $1252(0.1)$ & $58(45,71)$ & $0.5(0.3,1.6)$ & 12 & 6 & 268.2 \\
\hline Breast & $5918(0.6)$ & $51(44,61)$ & $4.7(1.7,5.8)$ & 6 & 5 & 23.3 \\
\hline Head and neck§ & $840(0.1)$ & $55(45,67)$ & $5.3(2.5,11.3)$ & $<5$ & $<5$ & 31.3 \\
\hline Gastrointestinal & $5688(0.6)$ & $65(54,75)$ & $1.8(1.1,5.1)$ & 17 & 14 & 107.9 \\
\hline Genitourinary & $1742(0.2)$ & $63(49,75)$ & $0.9(0.9,0.9)$ & $<5$ & $<5$ & 15.5 \\
\hline Neural & $457(0.1)$ & $55(45,71)$ & $2.1(2.1,2.1)$ & $<5$ & $<5$ & 94.5 \\
\hline Other/Unknown & $1890(0.2)$ & $53(41,69)$ & $1.3(0.4,7.0)$ & $<5$ & $<5$ & 48.6 \\
\hline Any cancer & $29448(2.9)$ & $57(45,70)$ & $1.6(0.4,4.9)$ & 91 & 60 & 83.3 \\
\hline
\end{tabular}

*Includes incident cases only.

†Among people diagnosed with active TB on or after their cancer diagnosis date, up to 5 years after the cancer diagnosis date. $\ddagger$ Person-years at-risk includes up to 5 years after cancer diagnosis.

$\S$ Excludes thyroid cancers.

TB, tuberculosis.

\section{DISCUSSION}

Our province-wide, retrospective cohort study demonstrates that migrants with cancer are at higher risk for active TB than migrants without cancer, with TB risk varying by tumour type. The highest risk of TB was observed among migrants with lung cancer, followed by sarcoma, leukaemia, lymphoma and GI malignancies. $\mathrm{HN}$, neural, skin, prostate and breast cancers all had an elevated point estimate for TB risk, however, the increase was not significant in multivariable analysis. Notably, the majority of active TB cases were clustered immediately before or after cancer diagnosis, but an appreciable number of people developed TB $>6$ months post-cancer diagnosis. This represents a population of people with potentially preventable TB.

To our knowledge, our study is the first to provide risk estimates for TB due to various cancer types in a cohort of migrants within a low incidence region. As latent TB screening of all immigrants has previously been shown to be economically challenging, understanding the selective impact of various malignancies becomes crucial to help clinicians identify migrants at highest risk of active TB.

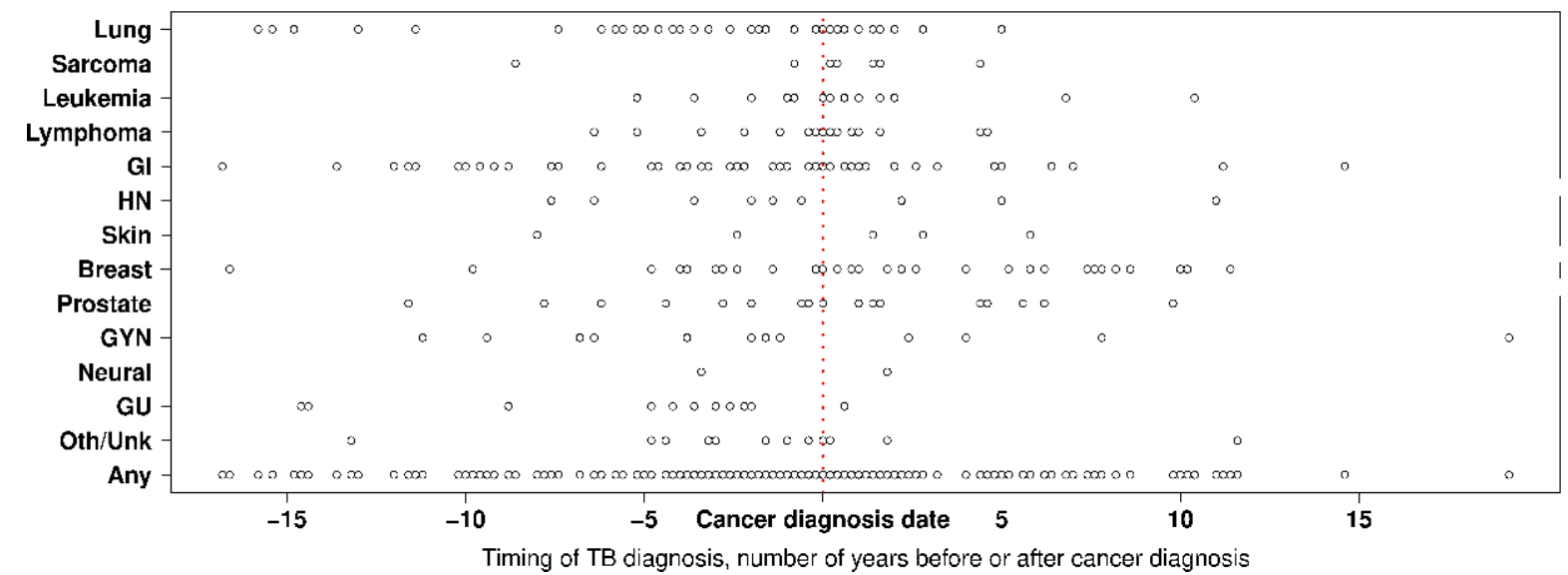

Figure 1 Timing of active TB diagnoses, number of years before and after cancer diagnoses (Gl, gastrointestinal; GYN, gynaecological; GU, genitourinary; HN, head and neck; Oth/Unk, other and unknown; ${ }^{\circ}$, active tuberculosis (TB) diagnosis.) 
Active TB

Cancer

Lung

Sarcoma

Leukemia

Lymphoma

GI

Neural

HN

Breast

Prostate

Skin

GYN

GU

Other

Any

\section{(cancer)}

25

5

11

12

17

$<5$

$<5$

6

10

$<5$

$<5$

$<5$

$<5$

91

\section{Active TB}

(without cancer) $\quad$ HR $(95 \%$ CI)

$2560 \quad 11.2(7.4,16.9)$

2580

$8.1(3.3,19.5)$

2574

$5.6(3.1,10.2)$

2573

$4.9(2.7,8.7)$

2568

$2.7(1.7,4.4)$

2584

$2.5(0.3,18.7)$

2582

$1.1(0.2,7.9)$

1258

$1.2(0.5,2.6)$

1310

$1.2(0.6,2.2)$

2583

$1.1(0.2,4.6)$

1262

$0.5(0.1,2.1)$

2584

$0.3(0.0,2.4)$

2584

$1.2(0.4,3.8)$

$2.5(2.0,3.1)$
Significant strengths of our study include large population cohort, risk estimates adjusted for other commonly recognised TB risk factors, stratification of TB risk by cancer type and long follow-up time. Given the wide capture of immigrant population over a 19-year follow-up period, we believe that our results may be applicable at other low incidence areas including other Canadian provinces, and regions within the USA.

The elevated TB risk observed in immigrants with lung and haematological cancers are in concordance with those noted in the non-immigrant populace as reported by Cheng et al, Simonsen et al and Shu et al, with some variations which may reflect differences in study populations and cancer treatments among different regions. ${ }^{10} 3435$ The mechanism through which malignancies increases TB risk is speculated to be multifactorial, including immunosuppression by both local and systemic effects. For lung cancer, mass effect and inflammation may disrupt local barriers to infection, increasing TB risk. ${ }^{34}$ Furthermore, infiltration of dormant TB granulomas by local tumour peptides in lung cancer may further increase reactivation risk. ${ }^{36}$ Importantly, our observed TB risk among lung cancer may be influenced by shared lifestyle variables, including smoking and alcohol use, which were not accounted for in our study. ${ }^{37-39}$ For haematological malignancies, immunosuppression may be due 
Table 3 TB risk in people diagnosed with lung cancer, univariable and multivariable HRs

\# TB cases per 100000 person-years Univariate HR $(95 \% \mathrm{Cl}) \quad$ Multivariable $\mathrm{HR}(95 \% \mathrm{Cl})$

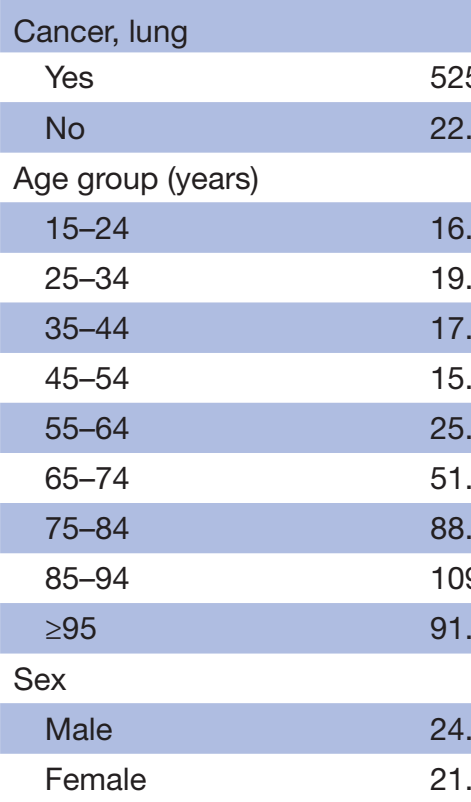

$\begin{array}{lll}525.1 & 25.2(17.0 \text { to } 37.6) & 11.2(7.4 \text { to } 16.9) \\ 22.4 & 1.0 & 1.0\end{array}$

$16.5 \quad 1.0 \quad 1.0$

$9.4 \quad 1.4(1.2$ to 1.6$) \quad 1.1(1.0$ to 1.3$)$

$17.0 \quad 1.2(1.1$ to 1.4$) \quad 1.1(1.0$ to 1.3$)$

$15.5 \quad 1.3(1.1$ to 1.5$) \quad 1.2(1.0$ to 1.4$)$

$2.1(1.8$ to 2.5$) \quad 1.7(1.4$ to 2.0$)$

$51.5 \quad 4.2(3.5$ to 4.9$) \quad 3.0$ (2.5 to 3.6$)$

$88.7 \quad 7.7(6.5$ to 9.1$) \quad 5.6(4.6$ to 6.8$)$

$109.1 \quad 10.1(8.0$ to 12.8$) \quad 8.4$ (6.5 to 10.9$)$

$91.2 \quad 9.0(4.4$ to 18.2$) \quad 8.4(4.1$ to 17.2$)$

24.3

$1.1(1.1$ to 1.2$)$

$1.2(1.1$ to 1.2$)$

TB incidence in country of origin

$\begin{array}{llll}0-30 & 2.4 & 1.0 & 1.0 \\ 31-100 & 9.9 & 4.2(3.0 \text { to } 5.8) & 4.2(3.0 \text { to } 5.9) \\ 101-200 & 21.0 & 9.2(6.6 \text { to } 12.6) & 9.4(6.8 \text { to } 12.9) \\ >200 / 100000 \text { population } & 45.2 & 19.7(14.3 \text { to } 27.1) & 18.6(13.5 \text { to } 25.6)\end{array}$

Immigration type

\begin{tabular}{|c|c|c|c|}
\hline Refugee & 29.8 & 2.1 (1.9 to 2.4$)$ & 2.0 (1.7 to 2.3$)$ \\
\hline Family & 33.5 & 2.4 (2.2 to 2.6$)$ & $1.2(1.1$ to 1.4$)$ \\
\hline Economic & 14.4 & 1.0 & 1.0 \\
\hline \multicolumn{4}{|l|}{ Years in $\mathrm{BC}$} \\
\hline $0-5$ & 29.8 & 1.4 (1.3 to 1.7$)$ & $1.6(1.4$ to 1.9$)$ \\
\hline$>5$ & 19.8 & 1.0 & 1.0 \\
\hline \multicolumn{4}{|c|}{ Active TB contact } \\
\hline Yes & 298.5 & 12.8 (9.0 to 18.4$)$ & $9.0(6.2$ to 12.9$)$ \\
\hline No & 22.4 & 1.0 & 1.0 \\
\hline \multicolumn{4}{|l|}{ HIV } \\
\hline Yes & 442.8 & 21.3 (16.1 to 28.2$)$ & 20.3 (15.1 to 27.2$)$ \\
\hline No & 22.2 & 1.0 & 1.0 \\
\hline \multicolumn{4}{|c|}{ Dialysis-dependent end-stage kidney disease } \\
\hline Yes & 553.6 & 27.1 (20.0 to 36.8$)$ & $8.2(5.9$ to 11.5$)$ \\
\hline No & 22.3 & 1.0 & 1.0 \\
\hline \multicolumn{4}{|c|}{ Immune-suppression* } \\
\hline Yes & 253.5 & 12.5 (10.3 to 15.3$)$ & 7.0 (5.6 to 8.6$)$ \\
\hline No & 21.8 & 1.0 & 1.0 \\
\hline \multicolumn{4}{|l|}{ Diabetes } \\
\hline Yes & 48.0 & 2.7 (2.4 to 3.0 ) & 1.3 (1.2 to 1.5$)$ \\
\hline No & 20.5 & 1.0 & 1.0 \\
\hline
\end{tabular}

*Includes treatment with immune-suppressing drugs (tumour necrosis factor- $\alpha$ inhibitors, disease-modifying antirheumatic drugs or high-dose steroids) or post-transplant. 
to multiple factors including bone marrow infiltration, impaired T-cell mediated immunity and superimposed chemotherapy. ${ }^{40-42}$ Although we were unable to account for chemotherapy regimens, sensitivity analyses examining effect of radiotherapy did not reveal any significant impact on active TB risk within our cohort.

Importantly, in contrast to previously assessed TB risk estimates within the general population, we found that HN cancer (excluding thyroid cancers) within the immigrant population resulted in a non-statistically significant elevated TB risk. The low incidence of HN cancer within our cohort resulted in wide CIs. We suspect that our observed lower TB risk among HN malignancies may reflect evolution in treatment and care of $\mathrm{HN}$ cancer, which may secondarily lower TB risk by reducing systemic immunosuppression and malnutrition. This includes transition to directed beam therapy, improved nutritional supplementation and closer surveillance. ${ }^{43}$ This is consistent with the systematic review conducted by Cheng et $a l,{ }^{10}$ which described a decrease in TB incidence among HN cancers after 1980 compared with pre-1980 period. In addition to changes in treatments, they speculated that decreased TB incidence may also reflect changes in the underlying aetiology of $\mathrm{HN}$ cancers, from non-Human papillomavirus (HPV)-associated HN cancer, which are typically related to heavy smoking and alcohol use, to HPV-related HN malignancies. ${ }^{104}$

Furthermore, to our knowledge, this study is the first to show increased adjusted TB risk in individuals with sarcomas. The link between sarcoma and TB has been described in rare case reports, and remains poorly understood. ${ }^{44}{ }^{45}$ While our risk estimates account for concurrent immunosuppression in patients with sarcoma, namely HIV and post-transplant state, we were unable to adjust for chemotherapy which may result in the observed elevated risk of active TB among individuals with sarcoma. Additionally, similar to HN cancers, the low incidence of sarcoma within our cohort accounts for the wide CIs observed. Literature also remains sparse on the association of GI malignancies and TB. While other authors have shown increased risk of TB with aerodigestive cancers, ${ }^{3446}$ our study shows an elevated active TB risk among GI malignancies independent from lung and HN cancers. After further stratifying GI cancer subtypes into smoking-associated (including oesophageal and gastric) and non-smoking-associated GI cancers, we did not observe significant difference in active TB risk (online supplemental table 1). It is speculated that concurrent comorbidities including significant weight loss and malnutrition may result in increased susceptibility to TB reactivation among GI cancers. ${ }^{447}$

The clustering of active TB around time of cancer diagnoses as noted in figure 1 may point to higher susceptibility to TB among patients with cancer, either by primary acquisition or latent $\mathrm{TB}$ reactivation. As mentioned, early identification and treatment of LTBI in high-risk patients with cancer is crucial to prevent active TB and the possibility of ongoing transmission, most importantly in oncology clinics and inpatient services. ${ }^{48}$ We observed that among cancers noted to increase active TB risk, including sarcoma, leukaemia, lymphoma, GI malignancies, an appreciable proportion of migrants were diagnosed with active $\mathrm{TB}>6$ months post-cancer diagnosis. These people represent potentially preventable TB as they could benefit from LTBI screening and treatment at the time of cancer diagnosis. In contrast to other malignancies, a lower proportion (approximately one-third) of active TB in immigrants with lung cancer was diagnosed $>6$ months post-cancer diagnosis. This phenomenon has been described by other authors and may be explained by the similar care pathways; TB can often produce masses or nodules which can imitate neoplasms in the lung, and therefore, similar imaging and diagnostic strategies involved for workup of both entities may explain this close association of lung cancer with TB. ${ }^{449-51}$ At present, in $\mathrm{BC}$, province-wide screening and treatment for LTBI in patients with cancer is not routine. Our data provide strong support for LTBI testing among migrants with sarcoma, leukaemia, lymphoma, lung and GI cancers.

\section{Limitations}

Our study has several limitations. First, we did not have access to smoking history, alcohol use and specific chemotherapeutic regimens, all of which increase active TB risk. ${ }^{3437-39}$ Certainly, the lack of smoking history likely confounds the relationship between smoking-related malignancies (ie, HN, lung cancer) and active TB risk. In a systematic review of smoking and $\mathrm{TB}$ risk, active smokers had a pooled risk estimate of 2.66 (95\% CI 2.15 to 3.28) for TB disease compared with non-smokers; this falls below the lower bounds of our 95\% CI on multivariate analysis for lung cancer. ${ }^{52}$ Therefore, smoking alone is unlikely to be the sole driver of TB risk in people with lung cancer.

Second, we were unable to account for contact to TB outside of BC, however, we expect these numbers to be low, given the overall low TB incidence rate in Canada. Third, the high rate of active TB in patients with cancer noted in our study may reflect the high level of care which patients with cancer require. While frequent follows ups for patients with cancer may lead to earlier diagnosis of TB by clinicians, the frequent visits to different healthcare by patients with cancer may result in varied exposure to TB based on specific hospitals and local city prevalence. However, we do believe that endemic spread is uncommon in BC, as shown by prior genomic epidemiology studies. ${ }^{53}$ Lastly, while our study population is large, the overall low number of active $\mathrm{TB}$ results in limited statistical power of our analyses.

Importantly, we conducted sensitivity analysis to account for individuals screened for LTBI and this did not reveal any significant differences in our observed risk estimates (online supplemental table 2). Further sensitivity analysis examining for effect modification by TB incidence in country of birth was performed (online supplemental table 3). The relationship between cancer subtype and 
TB risk varied between low and high incidence regions, but the overlap in CIs makes the data difficult to interpret further. Lastly, among immigrants with different malignancies, varying presentations of active TB (pulmonary and extrapulmonary) were noted. While lung cancer and lymphoma exhibited higher pulmonary TB diagnoses, limited numbers of active TB among other malignancies make it challenging to further discern relationship between cancer and type of TB presentation (online supplemental table 4). Despite our stated limitations in understanding the causal role of malignancy in TB risk, we do note that people with certain malignancies have high rates of active $\mathrm{TB}$, and may potentially benefit from LTBI screening and treatment, regardless of the underlying causal pathway.

\section{CONCLUSION}

In people that migrate to $\mathrm{BC}$, specific cancers increase active TB risk, with highest risk noted in lung, sarcoma, leukaemia, lymphoma and GI malignancies. An appreciable number of active TB cases are diagnosed $>6$ months after cancer diagnoses and may be potentially preventable with vigilant LTBI screening and therapy. While current Canadian TB guidelines recommend LTBI screening in migrants with haematological or $\mathrm{HN}$ cancer, our data provide evidence of the preventable burden of active TB in migrants with other malignancies who could be considered for LTBI screening and therapy.

Contributors All authors contributed equally to study conception, manuscript drafting and critical review, data analyses and provided final approval of the manuscript. JJ and VC were involved in study design, funding acquisition, statistical analysis, critical revision and supervision of the project. DSK, LR, KR, HS and CR participated in manuscript drafting, statistical analysis, critical revision and data interpretation.

Funding Michael Smith Foundation for Health Research and Canadian Institutes for Health Research (Grant: 377364).

Disclaimer All inferences, opinions and conclusions drawn in this study are those of the authors, and do not reflect the opinions or policies of the data steward(s).

Competing interests None declared.

Patient consent for publication Not required.

Ethics approval This study received ethical approval from the University of BC Clinical Ethics Review Board (H16-00625).

Provenance and peer review Not commissioned; externally peer reviewed.

Data availability statement Data may be obtained from a third party and are not publicly available. Data were extracted as deidentified datasets from several linked health administrative databases, including a national immigration database (Population Data BC and IRCC), a provincial health insurance registry, hospitalisation, physician billing, outpatient pharmacy, vital statistics, HIV and endstage renal disease (ESRD) registries. Additional data were extracted from the BC Provincial TB and BC Cancer Agency registries. Data may be obtained on authorised request to the respective data stewards as detailed in the study protocol.

Supplemental material This content has been supplied by the author(s). It has not been vetted by BMJ Publishing Group Limited (BMJ) and may not have been peer-reviewed. Any opinions or recommendations discussed are solely those of the author(s) and are not endorsed by BMJ. BMJ disclaims all liability and responsibility arising from any reliance placed on the content. Where the content includes any translated material, BMJ does not warrant the accuracy and reliability of the translations (including but not limited to local regulations, clinical guidelines, terminology, drug names and drug dosages), and is not responsible for any error and/or omissions arising from translation and adaptation or otherwise.

Open access This is an open access article distributed in accordance with the Creative Commons Attribution Non Commercial (CC BY-NC 4.0) license, which permits others to distribute, remix, adapt, build upon this work non-commercially, and license their derivative works on different terms, provided the original work is properly cited, appropriate credit is given, any changes made indicated, and the use is non-commercial. See: http://creativecommons.org/licenses/by-nc/4.0/.

Author note The corresponding author (JJ) has full access to all data in the study and takes responsibility for the integrity of the data and accuracy of analysis.

ORCID iD

Divjot S Kumar http://orcid.org/0000-0001-7175-414X

\section{REFERENCES}

1 WHO World TB Database. Global tuberculosis report 2019, 2019. Available: https://www.who.int/tb/publications/global_report/en/

2 World Health Organization, End TB Strategy. Global strategy and targets for tuberculosis prevention, care and control after 2015. World Health Organization, 2015. Available: https://www.who.int/tb/ strategy/End_TB_Strategy.pdf?ua=1

3 Lönnroth K, Migliori GB, Abubakar I, et al. Towards tuberculosis elimination: an action framework for low-incidence countries. Eur Respir J 2015;45:928-52.

4 World Health Organization and European Respiratory Society. Framework towards TB elimination in low incidence countries, 2014. Available: https://www.who.int/tb/publications/Towards_TB_ Eliminationfactsheet.pdf

5 Campbell JR, Chen W, Johnston J, et al. Latent tuberculosis infection screening in immigrants to low-incidence countries: a meta-analysis. Mol Diagn Ther 2015;19:107-17.

6 Campbell J, Marra F, Cook V, et al. Screening immigrants for latent tuberculosis: do we have the resources? CMAJ 2014;186:246-7.

7 Pareek M, Watson JP, Ormerod LP, et al. Screening of immigrants in the UK for imported latent tuberculosis: a multicentre cohort study and cost-effectiveness analysis. Lancet Infect Dis 2011;11:435-44.

8 Public Health Agency of Canada. Canadian tuberculosis standards 7th edition. Ottawa: aem, 2014. https://www.canada.ca/en/ public-health/services/infectious-diseases/canadian-tuberculosisstandards-7th-edition.html

9 American Thoracic Society and Centers of Disease Control and Prevention. Targeted tuberculin testing and treatment of latent tuberculosis infection. Am J Respir Crit Care Med 2000;161:S221-47.

10 Cheng MP, Abou Chakra CN, Yansouni CP, et al. Risk of active tuberculosis in patients with cancer: a systematic review and metaanalysis. Clin Infect Dis 2017;64:ciw838.

11 Ronald LA, Campbell JR, Balshaw RF, et al. Predicting tuberculosis risk in the foreign-born population of British Columbia, Canada: study protocol for a retrospective population-based cohort study. BMJ Open 2016;6:e013488.

12 Statistics Canada. Population size and growth rate, Canada, provinces and territories, 2006 to 2011 and 2011 to 2016, 2017. Available: https://www150.statcan.gc.ca/n1/daily-quotidien/170208/ t002a-eng.htm

13 Statistics Canada. Number and proportion of foreign-born population in Canada, 1871 to 2036, 2017. Available: https://www.statcan.gc. $\mathrm{ca} / \mathrm{eng} / \mathrm{dai} / \mathrm{btd} /$ othervisuals/other006

14 Public Health Agency of Canada. Tuberculosis in Canada 2016 2018. Available: https://www.canada.ca/en/public-health/services/ reports-publications/canada-communicable-disease-reportccdr/monthly-issue/2018-44/issue-3-4-march-1-2018/article-1tuberculosis-2016.html

15 Population Data BC. Population data BC, 2016. Available: https:// www.popdata.bc.ca

16 Government of Canada. Immigration statistics Canada, 2016. Available: http://epe.lac-bac.gc.ca/100/202/301/immigration_ statistics-ef/index.html

17 Immigration, Refugees, and Citizenship Canada (IRCC) [creator]. Permanent resident database [Internet]. Population Data BC [publisher]. Data Extract. IRCC; 2015, 2014. Available: http://www. popdata.bc.ca/data

18 Statistics Canada (2009) [creator]. Statistics Canada income band data. Catalogue number: 13C0016 [Internet]. Population data BC [publisher]. Data Extract, 2014. Available: http://www.popdata.bc.ca/ data 
19 Canadian Institute for Health Information [creator] (2015). Discharge abstract database (Hospital Separations) [Internet]. Population Data BC [publisher]. Data Extract. MOH, 2014. Available: http://www. popdata.bc.ca/data

20 BC renal agency [creator] (2015). BC Provincial Renal Agency Database (PROMIS) [Internet]. Population Data BC [publisher]. Data Extract. PROMIS, 2014. Available: http://www.popdata.bc.ca/data

21 BC Vital Statistics Agency [creator] (2015). BC Vital Statistics Deaths [Internet]. Population Data BC [publisher]. Data Extract. BC Vital Statistics Agency, 2014. Available: http://www.popdata.bc.ca/data

22 British Columbia Ministry of Health [creator] (2015). Consolidation File (MSP Registration \& Premium Billing) [Internet]. Population Data BC [publisher]. Data Extract. MOH, 2015. Available: http://www. popdata.bc.ca/data

23 BC Ministry of Health [creator] (2015). PharmaNet [Internet]. BC Ministry of Health Data Stewardship Committee [publisher]. Data Extract. MOH Data Stewardship Committee, 2014. Available: http:// www.popdata.bc.ca/data

24 BC Centre for Disease Control [creator] (2015). BC Provincial HIV/ AIDS Surveillance Database (BCCDC-HAISIS) [Internet]. Population Data BC [publisher]. Data Extract. BCCDC, 2014. Available: http:// www.popdata.bc.ca/data

25 BC Centre of Disease Control [creator] (2015). BC Provincial TB Registry (BCCDC-iPHIS). Population Data BC [publisher] Data Extract BCCDC [Internet], 2014. Available: http://www.popdata.bc. $\mathrm{ca} /$ data

26 BC Cancer Agency. Bc cancer registry, 2016. Available: http://www. bccancer.bc.ca/health-professionals/professional-resources/bccancer-registry

27 BC Cancer Agency [creator] (2015). BC Cancer Agency Registry Data [Internet]. Population Data BC [publisher]. Data Extract. BCCA, 2014. Available: http://www.popdata.bc.ca/data

28 British Columbia Ministry of Health [creator] (2015). Medical Services Plan (MSP) Payment Information File [Internet]. Population Data BC [publisher]. Data Extract. MOH, 2014. Available: http://www.popdata. bc.ca/data

29 Ministry of Health. MSP for B.C. Residents - Province of British Columbia [Internet], 2016. Available: https://www2.gov.bc.ca/gov/ content/health/health-drug-coverage/msp/bc-residents

30 Public Health Agency of Canada. Canadian tuberculosis reporting system: reporting form completion guidelines, version 1.9, 2011. Available: http://www.phac-aspc.gc.ca/tbpc-latb/pdf/guidelinesformeng.pdf

31 WHO. WHO world TB database, 2016. Available: http://www.who.int/ tb/country/data/download/en/

32 Ronald LA, Campbell JR, Rose C, et al. Estimated impact of World Health organization latent tuberculosis screening guidelines in a region with a low tuberculosis incidence: retrospective cohort study. Clin Infect Dis 2019;69:2101-8.

33 Thomas P, Foley R, Kosowicz L. Reactivation of pulmonary tuberculosis following local radiation therapy of prostate cancer. Conn Med 2014;78:77-80.

34 Simonsen DF, Farkas DK, Horsburgh CR, et al. Increased risk of active tuberculosis after cancer diagnosis. J Infect 2017;74:590-8.
35 Shu C-C, Liao K-M, Chen Y-C, et al. The burdens of tuberculosis on patients with malignancy: incidence, mortality and relapse. Sci Rep 2019;9:11901.

36 Suzuki Y, Imokawa S, Sato J, et al. Cumulative incidence of tuberculosis in lung cancer patients in Japan: a 6-year observational study. Respir Investig 2016;54:179-83.

37 Maurya V, Vijayan VK, Shah A. Smoking and tuberculosis: an association overlooked. Int J Tuberc Lung Dis 2002;6:942-51.

38 den Boon S, van Lill SWP, Borgdorff MW, et al. Association between smoking and tuberculosis infection: a population survey in a high tuberculosis incidence area. Thorax 2005;60:555-7.

39 Rehm J, Samokhvalov AV, Neuman MG, et al. The association between alcohol use, alcohol use disorders and tuberculosis (TB). A systematic review. BMC Public Health 2009;9:450.

40 Advani SH, Banavali SD. Pattern of infection in hematologic malignancies: an Indian experience. Rev Infect Dis 1989;11 Suppl 7:S1621-8.

41 Au WY, Kwong YL, Ma SK, et al. Hairy cell leukemia in Hong Kong Chinese: a 12-year retrospective survey. Hematol Oncol 2000;18:155-9.

42 Liu C-J, Hong Y-C, Teng C-J, et al. Risk and impact of tuberculosis in patients with chronic myeloid leukemia: a nationwide populationbased study in Taiwan. Int J Cancer 2015;136:1881-7.

43 Marur S, Forastiere AA. Head and neck squamous cell carcinoma: update on epidemiology, diagnosis, and treatment. Mayo Clin Proc 2016:91:386-96.

44 Kaplan $\mathrm{MH}$, Armstrong D, Rosen P. Tuberculosis complicating neoplastic disease. A review of 201 cases. Cancer 1974;33:850-8.

45 Aggarwal R, Rao S. Coexistence of tuberculosis with histiocytic sarcoma: a rare association. J Cancer Res Ther 2015;11:658.

46 Wu C-Y, Hu H-Y, Pu C-Y, et al. Aerodigestive tract, lung and haematological cancers are risk factors for tuberculosis: an 8-year population-based study. Int J Tuberc Lung Dis 2011;15:125-30.

47 Vento S, Lanzafame M. Tuberculosis and cancer: a complex and dangerous liaison. Lancet Oncol 2011;12:520-2.

48 Parry C, Kent EE, Mariotto AB, et al. Cancer survivors: a booming population. Cancer Epidemiol Biomarkers Prev 2011;20:1996-2005.

49 Chen C-Y, Wang J-Y, Chien Y-C, et al. Lung cancer mimicking pulmonary tuberculosis in a TB-endemic country: the role of early invasive diagnostic procedures. Lung Cancer Manag 2015;4:9-16.

50 Ciftci F, Shimbori N, Karnak D. Concurrent central nervous system and endobronchial tuberculosis mimicking a metastatic lung cancer. Clin Respir J 2017;11:98-102.

51 Davies PDO, Pai M. The diagnosis and misdiagnosis of tuberculosis [State of the art series. Tuberculosis. Edited by I. D. Rusen. Number 1 in the series]. The International Journal of Tuberculosis and Lung Disease 2008;12:1226-34.

52 Bates MN, Khalakdina A, Pai M, et al. Risk of tuberculosis from exposure to tobacco smoke: a systematic review and meta-analysis. Arch Intern Med 2007;167:335.

53 Guthrie JL, Kong C, Roth D, et al. Molecular epidemiology of tuberculosis in British Columbia, Canada: a 10-year retrospective study. Clin Infect Dis 2018;66:849-56. 